\title{
Modified DWT for Feature Extraction of Bear Failure Vibration Signal
}

\author{
Junjiang $\mathrm{Zhu}^{*}$ and Lingsong $\mathrm{He}$
}

Department of Mechanical Science and Engineering, Huazhong University of Science and Technology, Wuhan, Hubei, 430074, China

\begin{abstract}
In this paper, bear fault is automatically diagnosed by using pattern recognition. To improve the resolution of lower frequency part, we introduce scale factors to discrete wavelet composition (DWT). The modified DWT combined with high order cumulates are used for vibration signal feature extraction. Besides we use principle component analysis to reduce dimension of the feature data. This feature extraction method has a lower dimension and a higher resolution for lower frequency parts. Therefore it can not only reveal the characteristics of non-linear relationship between amounts of features, but also help to improve the speed and accuracy of classification. Finally neural network algorithm is used for fault classification. Result shows that our method can accurately and efficiently identify the type of bearing failures.
\end{abstract}

Keywords: Nonlinear vibration signal, neural network, PCA, wavelet.

\section{INTRODUCTION}

Bears, whose qualities affect the running state of the equipment, are important parts for many mechanical devices. Their faultiness could lead to abnormal vibration, moreover the damage of the devices. Therefore, it is very necessary to monitor diagnose the state of bears intelligently.

By using pattern recognition for fault diagnose contain mainly two steps: feature extraction and classification. Selected features could affect the reliability as well as the accuracy, which make it a research hot spot. Until now the features for bear fault diagnoses could be extracted for time domain, frequency domain or wavelet domain. In researches, features from time domain are proved to be most promised [1]. Effective value, peak value, pulse factor, kurtosis, margin factor, waveform factor [2-4] are widely used as time domain features. The method of extracting time domain features is extremely easy. Moreover, the time domain features are sensible for early failures. However, they are incapable for judging the place, type, and degree of the fault [5]. Faults in bears lead to periodic impulse. In this view, frequency domain features are believed to contain fault information. The impulse is usually be modulated and polluted by noise. Therefore usage of frequency could not cover all the fault information. Time-frequency parameters [6] contain both time-domain and frequency-domain information. However the resolution are fixed, therefore the lower frequency parts which carries important information could be ignored.

Wavelets decomposition offers resolution both in timedomain and frequency domain and adopts special sampling intervals to improve the resolution of details as to be widely used in fault diagnose [7]. Wavelet coefficients represent fault features are high dimensional, if directly be fed to classifier could increase the computational burden.

The vibration signal under faulted bear are nonlinear, therefore should be processed with nonlinear transform. In this paper we combined discrete wavelet decomposition and high order cumulates for feature extraction, and then the features are compressed by principle component analysis before fed to the classifier. By this way the fault can be classified automatically.

\section{FEATURE EXTRACTION}

\subsection{Modified Discrete Wavelet Decomposition}

Wavelet composition offers localization in both time domain and frequency domain, and analyzes signals from a multi-scale view, therefore is more suitable for nonlinear feature extraction. A wavelet function can be expressed as in Eql (1):

$\psi a, b(t)=\frac{1}{\sqrt{a}} \psi\left(\frac{t-b}{a}\right)$

where, $a$ refers to the scale of a wavelets and $b$ represents the bias. For the continue wavelet transform, a serial of coefficients are generated under the inner product between the signal and wavelets by changing the scale and bias of wavelets. However, in order to extract the features from a digital signal, we use discrete wavelet decomposition. A standard binary discrete wavelet can be expressed as in Eql (2):

$\psi_{m, n}(t)=2^{-m / 2} \psi\left(2^{-m} t-n\right)$

The binary discrete wavelet is mostly used because it has both decomposition and reconstruction algorithms. For the standard discrete wavelet decomposition process can be expressed as in Fig. (1). 


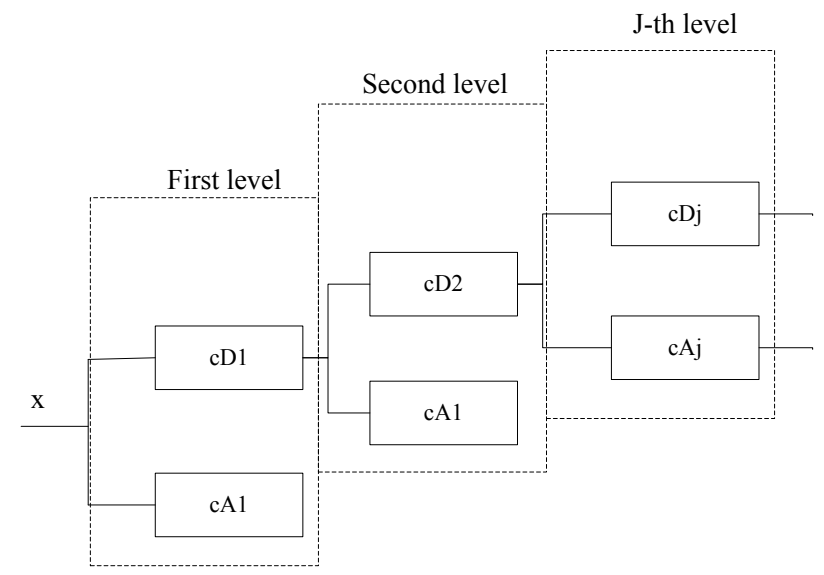

Fig. (1). Process of DWT.

When the faulted bear rolls in the high speed, a transient force will generate. The force will function on the spring damping system which is composed of a bearing and a bearing block. A broadband vibration signal is then generated. This signal is low frequency and believed as the fault feature. Therefore, the key problem is to design an appropriate filter. In traditional, the filter is mostly designed according to experience. DWT can make up the shortcoming of the traditional method. In DWT, each level of wavelets decomposition is realized by a low-pass and a high-pass filter. For standard DWT, both filters are fixed, that is to say, both the low-pass filter and high-pass frequency have a normalized cut-off frequency of $0.5 \mathrm{~Hz}$. Therefore resolution of high frequency and low frequency are the same, which leads it not suitable for analysis of high-require signals. As useful information for fault diagnosis is contained in the lower frequency parts, we present a modified DWT in this paper. The modified DWT is shown as Fig. (2). In Fig. (2), $\alpha$ represents scale factor of the low-pass frequency filter, and $\beta$ represents scale factor of the high-pass frequency filter, in order to underline the lower frequency part. By introducing the scale factor, the normalized cut-off frequency is changed to $0.5 /$ scale factor. In other words when the scale factor is more than 1 , the normalized cut-off frequency will less than $0.5 \mathrm{~Hz}$, means high resolution. In this paper we choose $\alpha=5 / 6$ and $\beta=0.5$.

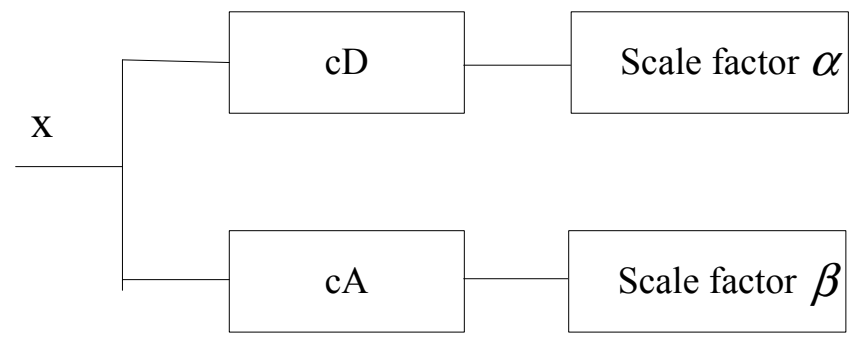

Fig. (2). Analysis filter bank.

\subsection{High Order Cumulates}

To reveal the nonlinear features, we adopt high order cumulates to statistic wavelets coefficients obtained by modified DWT. The high order cumulates are calculated as in $\operatorname{Eql}(3)$.

$$
\begin{aligned}
& C_{1}^{X}=E[X(n)] \\
& C_{2}^{X}(i)=E[X(n) X(n+i)] \\
& C_{3}^{X}(i, j)=E[X(n) X(n+i) X(n+j)] \\
& C_{4}^{X}(i, j, k)=E[X(n) X(n+i) X(n+j) X(n+k)] \\
& \quad-R_{x}(i) R_{x}(j-k)-R_{x}(j) R_{x}(k-j) \\
& \quad-R_{x}(k) R_{x}(i-j)
\end{aligned}
$$

where, the first and second order cumulates describe the center and the dispersion degree respectively. They just show the basic features of the signals. The third and the fourth order cumulates are used to describe the nonlinear features. Therefore in this paper we used the fourth order cumulates to extract fault features.

\subsection{Principle Component Analysis}

Using modified DWT and high order cumulates, we could obtain the nonlinear features. However the extracted features are of high dimension. As a result it will burden compute load and increase the accuracy, if we send the features into the classifier directly. In this paper we use principle component analysis to compress the features. PCA can visualize the contribution and during the process of mapping the high dimension data into low dimension data, only the features contribute more are kept.

Suppose there are $N \quad n$-dimensional samples $X_{k}=\left(x_{1}, x_{2}, \ldots x_{n}\right)^{T}$. The PCA process them as bellows:

(a) Get the mean value of all the samples

$$
X=\frac{1}{N} \sum_{k=1}^{N} X_{k}
$$

(b) Calculate the covariance matrix

$$
S=\frac{1}{N} \sum_{k=1}^{N}\left(X_{k}-X\right)\left(X_{k}-X\right)^{T}
$$

(c) Calculate the eigenvalue $\lambda_{1}, \lambda_{2}, \cdots \lambda_{n}$ and the eigenvector $v_{1}, v_{2}, \cdots v_{n}$ of the the covariance matrix.

(d) Sort the eigenvalue, the larger eigenvalue contributes more.

(e) Select the first $m$ components and map the features into a lower dimensional space:

$Y=V^{T} X_{k}$

where, $V=\left(\nu_{1}, \nu_{2}, \cdots \nu_{m}\right)$

\section{CLASSIFIER}

Neural network is a learning system with ability of adaptive and self-organizing. Hence it is widely used to reveal the relationship between features from vibration signals and the type of the fault. BP neural network is of strong learning ability, extension and generalization $[8,9]$. Therefore we use BP neural network as classifier. For a multi-layer neural network, increasing the number of net 
cannot improve the accuracy obviously, therefore we design a three layer feed neural network contains input layer, hidden layer and output layer, as shown in Fig. (3).

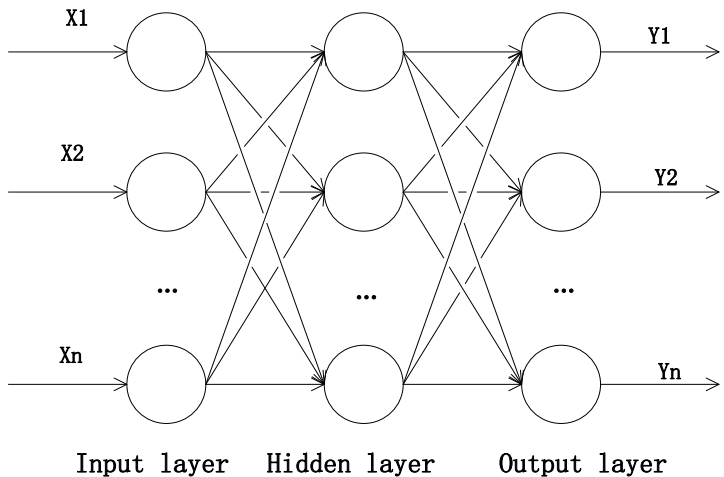

Fig. (3). The structure of a three layer neural network.

\section{EXPERIMENT}

The data of this paper comes from the bear data center of case Western Reserve University. The model of bears are $6205-2 R S$ deep groove ball bearing from SKF. The types of fault include inner race single point fault, outer raceway fault and pitting fault of ball. The fault diameter and depth are $0.1778 \mathrm{~mm}$ and $0.2794 \mathrm{~mm}$ respectively. During the experiment, the loads are 0HP, $1 \mathrm{HP}, 2 \mathrm{HP}$ and $3 \mathrm{HP}$ with speeds of $1797 \mathrm{r} / \mathrm{min}, 1772 \mathrm{r} / \mathrm{min}, 1750 \mathrm{r} / \mathrm{min}$ and 1730 $\mathrm{r} /$ min. Digital data was collected using a 16 channel data recorder at 12,000 samples per second. We classify three types of the data, normal vibration signals, inner race fault vibration signals and outer raceway fault vibration signals.

Table 1. Statics of the features.

\begin{tabular}{|c|c|c|c|}
\hline Record & Inner Race & Outer Race & Normal \\
\hline \hline 1 & $0.0316 \pm 0.0168$ & $0.0843 \pm 0.0848$ & $0.0162 \pm 0.0034$ \\
\hline 2 & $0.0110 \pm 0.0064$ & $0.0017 \pm 0.0356$ & $0.0055 \pm 0.0031$ \\
\hline 3 & $0.0002 \pm 0.0061$ & $-0.0018 \pm 0.0129$ & $0.0234 \pm 0.0021$ \\
\hline 4 & $0.0060 \pm 0.0039$ & $-0.0042 \pm 0.0082$ & $0.0101 \pm 0.0037$ \\
\hline 5 & $0.0034 \pm 0.0033$ & $-0.003 \pm 0.004$ & $0.0903 \pm 0.0061$ \\
\hline 6 & $0.0235 \pm 0.0013$ & $-0.022 \pm 0.007$ & $0.0581 \pm 0.0011$ \\
\hline 7 & $0.0018 \pm 0.0006$ & $-0.019 \pm 0.0107$ & $0.0713 \pm 0.0126$ \\
\hline
\end{tabular}

The vibration data is cut to pieces with length of 2048 . We use 18 kinds of wavelets to process the pieces. They are 2-8 order Haar wavelets, 2-7 order Daubenchies wavelets and 2-6 order Symlet wavelets.

By using the second, third and fourth order cumulates of the first 8 nodes from the coefficients, we obtain 24 features. Then the 24 features are compressed by PCA to 7 features. Table 1 list the statistics of feature data handled by present method using fourth order Daubenchies wavelets.

We code normal vibration signals, inner race fault and outer raceway fault with $[0,0,1],[0,1,0]$ and $[1,0,0]$. The coding matrix and selected features are sent into designed classifier. The activation function is selected as Sigmoid. The training method is BP algorithm. Fig. (4) shows the training result.

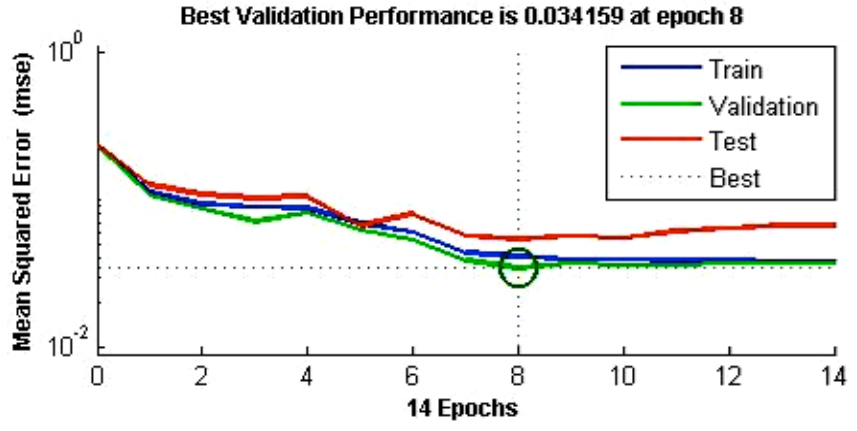

Fig. (4). Training result of neural network.

The results are given by comparing standard DWT and modified DWT under different wavelets, as shown in Table 2.

Table 2 shows that by using modified DWT we can get much higher accuracy when compared to the standard DWT. We can also see that by using the Daubenchies family wavelets we can get the best results with accuracy of $96.48 \%$, specificity of $96.04 \%$ and sensitivity of $96.90 \%$.

\section{CONCLUSION}

We added scale factors to DWT, and used the modified DWT to extrude the nonlinear features in vibration signals. A scale factor changes cut-off frequency, as a result changes resolution. We set the scale factors less than 1 , therefore improve the resolution of the lower frequency parts. Then high order cumulates is imposed on wavelets coefficients to extract the features that related to the fault. We used PCA to map the extracted features into a lower dimension space, and

Table 2. Results of classification.

\begin{tabular}{|c|c|c|c|c|}
\hline & Wavelets & Accuracy (\%) & Specificity (\%) & Sensitivity (\%) \\
\hline \hline \multirow{3}{*}{ Standard DWT } & Haar & 78.58 & 86.38 & 74.25 \\
\cline { 2 - 5 } & Symlet & 82.17 & 81.24 & 80.51 \\
\cline { 2 - 5 } & Daubenchies & 87.28 & 89.09 & 90.14 \\
\hline \multirow{3}{*}{ Modified DWT } & Haar & 93.55 & 92.38 & 95.12 \\
\cline { 2 - 5 } & Symlet & 94.87 & 95.25 & 95.48 \\
\cline { 2 - 5 } & Daubenchies & 96.48 & 96.04 & 96.90 \\
\hline
\end{tabular}


then fed them to a neural network classifier. The result shows that by using Daubenchies we get best results, the accuracy could reach $96.48 \%$, while the specificity and sensitivity reach $96.04 \%$ and $96.90 \%$ retrospectively. It demonstrates superiority of present method, and besides the method for modifying DWT also provide ideas for processing other kinds of nonlinear signals.

\section{CONFLICT OF INTEREST}

The authors confirm that this article content has no conflict of interest.

\section{ACKNOWLEDGEMENTS}

This paper is supported by the 'Fundamental Research Funds for the Central Universities', (HUST:CXY12Q023) and the ministry of science and technology of the People's Republic of China, within the framework of the project the CNC products innovation demonstration (Contract number: 2012BAF13B06)

\section{REFERENCES}

[1] S. Wan, and M. Wu, "Fault diagnosis for rolling bearing based trend analysis of time domain parameters," Mechanical
Engineering \& Automation, no. 03, pp. 108-110+113, 2010. (in Chinese)

[2] J. Ma, and L. Luo, "A feature parameter selection method of rolling bearing fault diagnosis," Mechanical Science And Technology, vol. 15, no. 5, pp. 136-140, 1996. (in Chinese)

[3] S. Cui, "Application of time domain indexes for rolling bearing fault diagnosis," Mechanical Engineering \& Automation, vol. 2 , no. 1, pp. 101-1021, 2008. (in Chinese)

[4] S. Chen, "Time domain analysis technology and its application in diagnosis for fault of mechnical devices," Journal of Mechanical Transmission, vol. 31, no. 3, pp. 79-83+4, 2007. (in Chinese)

[5] B. Long, S.L. Tian, and H.J. Wang, "Diagnostics of filtered analog circuits with tolerance based on LS-SVM using frequency features," Journal Of Electronic Testing-Theory And Applications, vol. 28 , no. 3, pp. 291-300, 2012.

[6] H. Arabaci, and O. Bilgin, "Neural network classification and diagnosis of broken rotor bar faults by means of short time fourier transform," IMECS 2009: International Multi-Conference Of Engineers And Computer Scientists, vol. 1-2, pp. 219-223, 2009.

[7] H. Zhang, S. Wang, Q. Zhang, and G. Zhai, "Reaserch on rolling bear fault diagnosis based on wavelet package transform," Journal of Vibration and Shock, vol. 23, no. 4, pp. 129-132+155, 2004. (in Chinese)

[8] Y. Luo, S. Chen, X. He, "Alphanumeric character recognition based on bp neural network classification and combined features," International Journal Of Computational Intelligence Systems, vol. 6, no. 6, pp. 1108-1115, 2013.

[9] R.J. Wang, X. Feng, Y. Xia, “A back propagation artificial neural network prediction model of the gate freeze time for injection molded polypropylenes," Journal Of Macromolecular Science Part B-Physics, vol. 52, no.10, pp. 1414-1426, 2013. 